\title{
Free Energy Balance in Gyrokinetic Turbulence
}

\author{
A. Bañón Navarro, ${ }^{1, \text { a) }}$ P. Morel, ${ }^{1}$ M. Albrecht-Marc, ${ }^{1}$ D. Carati, ${ }^{1}$ F. Merz, ${ }^{2}$ T. Görler, ${ }^{2}$ and F. Jenko ${ }^{2}$ \\ ${ }^{1)}$ Statistical and Plasma Physics Laboratory, Université Libre de Bruxelles, Belgium \\ 2) Max-Planck-Institut für Plasmaphysik, Boltzmannstr. 2, D-85748 Garching, \\ Germany
}

Free energy plays an important role in gyrokinetic theory since it is known to be a nonlinear invariant. Its evolution equations are derived and analyzed for the case of ion temperature gradient driven turbulence, using the formalism adopted in the GENE code. In particular, the ion temperature gradient drive, the collisional dissipation as well as entropy/electrostatic energy transfer channels represented by linear curvature and parallel terms are analyzed in detail.

\section{INTRODUCTION}

Gyrokinetic simulations take advantage of the helical motion of charged particles in presence of intense magnetic fields to simplify the numerical study of magnetized plasmas. In particular, in the limit of low frequencies compared to the gyrofrequencies related to this helical motion, a five dimensional (instead of a six-dimensional) velocity-position distribution function ${ }^{1}$ can be used to describe the plasma. The reduction of the number of phase space dimensions as well as the elimination of small (and irrelevant) spatio-temporal scales are the major advantages of the gyrokinetic formalism in terms of numerical simulations.

As is well known, gyrokinetic theory - although represented by a quite complex set of nonlinear partial integrodifferential equations - possesses interesting analytical properties. Indeed, it has been shown that gyrokinetics has a nonlinear quadratic invariant playing a similar role as the kinetic energy in the Navier-Stokes turbulence. This quadratic invariant has been identified as the free-energy (see Ref. ${ }^{2}$ and various references therein). It has been shown in a recent Letter ${ }^{3}$ that the free energy dynamics exhibits a cascade regime ${ }^{4}$ in which injection in the large scales is due to imposed mean gradients of temperature or density and dissipation due to collisions is observed in the small scales. Moreover, a nonlinear conservative interaction has been shown to transfer free energy from the injection domain to the dissipation range.

The purpose of this paper is to study further this dynamics by investigating in details the free energy balance in gyrokinetic turbulence. The gyrokinetic equation is briefly summarized in section II. In section III, the global free energy balance is discussed from both theoretical and numerical viewpoints. In section IV, the energy balance is studied for each scale by using a Fourier representation that allows for a better understanding of the cascade processes, followed by a summarizing discussion in Section V.

\footnotetext{
a) Electronic mail: abanonna@ulb.ac.be
}

\section{GYROKINETIC MODEL}

The gyrokinetic formalism can be designed for an arbitrary number of charged particle species in various geometries. In the present study, however, the analysis is limited to the simple scenario of a single ion species and adiabatic electrons in the context of a large aspect-ratio, circular cross-section model equilibrium ${ }^{5}$. In this case the evolution equation for the ion distribution function $f_{i}$ appropriately normalized reads (for details, see Ref. ${ }^{6}$ ):

$$
\partial_{t} f_{i}=L\left[f_{i}\right]+N\left[f_{i}, f_{i}\right]+D\left[f_{i}\right],
$$

where the linear term can be split into three contributions, $L\left[f_{i}\right]=L_{G}\left[f_{i}\right]+L_{C}\left[f_{i}\right]+L_{\|}\left[f_{i}\right]$, with

$$
\begin{aligned}
L_{G}\left[f_{i}\right] & =-\left(\omega_{n i}+\left(v_{\|}^{2}+\mu B_{0}-3 / 2\right) \omega_{T i}\right) F_{0 i} \frac{\partial \bar{\phi}_{1}}{\partial y}, \\
L_{C}\left[f_{i}\right] & =-\frac{T_{0 i}\left(2 v_{\|}^{2}+\mu B_{0}\right)}{q_{i} B_{0}}\left(K_{x} \frac{\partial h_{i}}{\partial x}+K_{y} \frac{\partial h_{i}}{\partial y}\right), \\
L_{\|}\left[f_{i}\right] & =\frac{v_{T i}}{2}\left[\frac{h_{i}}{F_{0 i}}, F_{0 i}\right]_{z v_{\|}} .
\end{aligned}
$$

Here, $h_{i}$ is referred to as the nonadiabatic part of the distribution function, $h_{i}=f_{i}+q_{i} F_{0 i} \bar{\phi}_{1} / T_{0 i}$ where $q_{i}$ denotes the ion charge (normalized to the elementary charge $e$ ), $F_{0 i}$ the background distribution function, $\bar{\phi}_{1}$ the gyroaveraged electrostatic potential, $v_{T i}=\sqrt{2 T_{0 i} / m_{i}}$ the ion thermal velocity, $T_{0 i}$ the ion temperature (normalized to the electron temperature) and $m_{i}$ the ion mass. It is stressed here that, since the electrons are treated adiabatically, all the distribution functions $f_{i}$ and $h_{i}$ refer to the ions and, to simplify the notations, no subscript ' $i$ ' is added in the following. The equilibrium magnetic field is assumed to be expressed by $B=B_{0} B_{\text {ref }}$ where $B_{\text {ref }}$ is the reference magnetic field on the magnetic axis. Finally, the Poisson brackets are defined by $[f, g]_{a b}=\partial_{a} f \partial_{b} g-\partial_{b} f \partial_{a} g$.

The first linear term $L_{G}$ represents the influence of the fixed ion density $\left(\omega_{n i}\right)$ and temperature $\left(\omega_{T i}\right)$ gradients expressed in major radius $R$ units, the second linear term $L_{C}$ describes effects due to magnetic curvature, and the third linear term $L_{\|}$contains the parallel dynamics involving magnetic trapping as well as the linear Landau damping. The nonlinear term $N$ represents the effect 
of the self-consistent electric field in the $\vec{E} \times \vec{B}$ drift of charged particles,

$$
N[f, f]=\left[\bar{\phi}_{1}, f\right]_{x y} .
$$

In the present study, the numerical analysis of the gyrokinetic equation (1) is performed using the GENE code ${ }^{6-8}$. In this code, the dissipation term $D[f]$ is given by

$$
D[f]=-\left(a_{x} \partial_{x}^{n}+a_{y} \partial_{y}^{n}+a_{z} \partial_{z}^{n}+a_{v_{\|}} \partial_{v_{\|}}^{n}\right) f
$$

where typically $n=4$ is used, and the coefficients $a_{x}, a_{y}$, $a_{z}$, and $a_{v_{\|}}$can be adapted to a specific class of physical problems (for details see Ref. ${ }^{9}$ ). In the local version of GENE used here, the distribution functions $f$ and other quantities like the electrostatic potential $\phi_{1}$ are Fourier transformed in the radial $(x)$ and poloidal directions $(y)$. The $x$ and $y$ coordinates are thus replaced respectively by $k_{x}$ and $k_{y}$. The subscript ' $k$ ' has been added to label the Fourier modes. The gyrokinetic Poisson equation used to determine the self-consistent electrostatic field is usually expressed in terms of the Fourier modes:

$$
\begin{array}{r}
\frac{q_{i}^{2} n_{0 i}}{T_{0 i}}\left[1-\Gamma_{0}\left(b_{i}\right)\right] \phi_{1 k}+n_{0 e}\left(\phi_{1 k}-\left\langle\phi_{1}\right\rangle_{F S}\right)= \\
\pi q_{i} B_{0} n_{0 i} \int J_{0}(\lambda) f_{k} d v_{\|} d \mu
\end{array}
$$

where $\lambda^{2}=2 k_{\perp}^{2} \mu / B_{0}, b_{i}=v_{T i}^{2} k_{\perp}^{2} /\left(2 \Omega_{i}\right)$ while $n_{0 e}$ and $n_{0 i}$ are respectively the equilibrium electron and ion densities. The functions $J_{0}$ and $\Gamma_{0}\left(b_{i}\right)=\exp \left(-b_{i}\right) I_{0}\left(b_{i}\right)$ are, respectively, the Bessel and the scaled modified Bessel functions of order zero. Finally, $k_{\perp}$ is the perpendicular wave number and $\Omega_{i}$ is the ion cyclotron frequency. The angular brackets $\left\langle\phi_{1}\right\rangle_{F S}$ are used to represent the flux surface average of the electric potential.

\section{GLOBAL FREE ENERGY BALANCE}

The nonlinear term in Eq. (1) has the property that it conserves the free energy $\mathcal{E}^{2,10,11}$. Actually, in the simple case treated here and represented by the coupled gyrokinetic (1) and Poisson (3) equations, the free energy can be split into two parts $\mathcal{E}=\mathcal{E}_{f}+\mathcal{E}_{\phi}$ that are each conserved by the nonlinear term. The first part is quadratic in $f$ and can be understood as the thermodynamic entropy,

$$
\mathcal{E}_{f}=\int \mathrm{d} \Lambda \frac{T_{0 i}}{F_{0 i}} \frac{f^{2}}{2} .
$$

The evolution equation for $\mathcal{E}_{f}$ is readily obtained from the gyrokinetic equation for $\partial_{t} f$ and is simply given by:

$$
\frac{\partial \mathcal{E}_{f}}{\partial t}=\int \mathrm{d} \Lambda \frac{T_{0 i}}{F_{0 i}} f \partial_{t} f .
$$

The gradient term $L_{G}$ in the right hand side of the equation for $\partial_{t} f$ thus leads to a term $\mathcal{G}_{f}$ in the equation for $\partial_{t} \mathcal{E}_{f}$ that is readily expressed as:

$$
\mathcal{G}_{f}=\int \mathrm{d} \Lambda \frac{T_{0 i}}{F_{0 i}} f L_{G}[f] .
$$

The contributions to the equation for $\partial_{t} \mathcal{E}_{f}$ from the curvature term $\mathcal{L}_{C, f}$, the parallel term $\mathcal{L}_{\|, f}$ and the dissipation term $\mathcal{D}_{f}$ are defined similarly. The nonlinear term, as already mentioned, does not contribute to the equation for $\partial_{t} \mathcal{E}_{f}$. In the expressions (4-6), the integration over $\Lambda$ has to be understood as a phase-space integration defined by:

$$
\begin{aligned}
& \int \mathrm{d} \Theta=\int \mathrm{d} z \int \mathrm{d} v_{\|} \int \mathrm{d} \mu \pi B_{0} n_{0 i} \\
& \int \mathrm{d} \Lambda=\int \mathrm{d} x \int \mathrm{d} y \int \mathrm{d} \Theta
\end{aligned}
$$

The second term in $\mathcal{E}$ is proportional to the product of $f$ and $\overline{\phi_{1}}$ :

$$
\mathcal{E}_{\phi}=\int \mathrm{d} \Lambda q_{i} \frac{\overline{\phi_{1}} f}{2} .
$$

It is usually referred to as the electrostatic energy. It should be noted that, owing to the Poisson equation (3), this second term (9) is also formally quadratic in $f$ and it can be shown that its time derivative is simply expressed by

$$
\frac{\partial \mathcal{E}_{\phi}}{\partial t}=\int \mathrm{d} \Lambda q_{i} \bar{\phi}_{1} \partial_{t} f .
$$

Again, using the explicit decomposition of $\partial_{t} f$ in terms of the linear, nonlinear and dissipation term, it is easy to define the expressions for the contribution of these terms to the electrostatic energy evolution equation $\left(\mathcal{G}_{\phi}, \mathcal{L}_{C, \phi}\right.$, $\left.\mathcal{L}_{\|, \phi}, \mathcal{D}_{\phi}\right)$. Again, the nonlinear term does not contribute to the equation for $\partial_{t} \mathcal{E}_{\phi}$. For instance, the curvature term in the electrostatic energy equation is given by:

$$
\mathcal{L}_{C, \phi}=\int \mathrm{d} \Lambda q_{i} \bar{\phi}_{1} L_{C}[f] .
$$

All these terms have different impact on the balance of $\mathcal{E}_{f}$ and $\mathcal{E}_{\phi}$. The parallel $L_{\|}$linear term can be shown to conserve the total free energy $\mathcal{E}$ but not the entropy and the electrostatic energy individually. Hence, the contributions of these terms to the balance equations satisfy the constraint $\mathcal{L}_{\|, \phi}=-\mathcal{L}_{\|, f}$. The curvature $L_{C}$ presents exactly the same property and, consequently, $\mathcal{L}_{C, \phi}=-\mathcal{L}_{C, f}$. The gradient terms can be shown to conserve the electrostatic energy $\left(\mathcal{G}_{\phi}=0\right)$ but not the entropy $\left(\mathcal{G}_{f} \neq 0\right)$. Finally, the dissipation terms $\mathcal{D}_{f}$ and $\mathcal{D}_{\phi}$ are non zero in both the entropy and the electrostatic energy equations. Taking into account all these properties, the entropy and the electrostatic energy balance equations can be written as:

$$
\frac{\partial \mathcal{E}_{f}}{\partial t}=\mathcal{G}_{f}+\mathcal{L}_{C, f}+\mathcal{L}_{\|, f}-\mathcal{D}_{f}
$$


and

$$
\frac{\partial \mathcal{E}_{\phi}}{\partial t}=\mathcal{L}_{C, \phi}+\mathcal{L}_{\|, \phi}-\mathcal{D}_{\phi}=-\mathcal{L}_{C, f}-\mathcal{L}_{\|, f}-\mathcal{D}_{\phi} .
$$

The total free energy balance equation is then given by the sum of these two relations:

$$
\frac{\partial \mathcal{E}}{\partial t}=\mathcal{G}-\mathcal{D}
$$

where $\mathcal{D}=\mathcal{D}_{f}+\mathcal{D}_{\phi}$. These properties have been checked numerically by considering the classical test-case of collisionless ion temperature gradient (ITG) turbulence usually referred to as the Cyclone Base Case ${ }^{12}$. The simulation domain is about 125 ion gyroradii wide in the perpendicular directions, and $128 \times 64 \times 16 \times 32 \times 8$ grid points are used in $\left(x, y, z, v_{\|}, \mu\right)$ space. Before analyzing the free energy balance equations in details, the numerical accuracy of the code has been checked by considering two simple tests. Firstly, the impact of the nonlinear term on the free energy balance $\mathcal{N}$ has been measured. Analytically this term should vanish exactly for all times. In practice, the ratio $\mathcal{N} / \overline{\mathcal{D}}$ can be used to assess the accuracy of the various algorithms used in GENE to discretize the gyrokinetic equation, both in the real space as in the velocity space. Here, $\overline{\mathcal{D}}$ is the time-averaged value of the dissipation which is very stable. Secondly, the residual $\Delta=\left(\frac{\partial \mathcal{E}}{\partial t}-\mathcal{G}-\mathcal{D}\right) / \overline{\mathcal{D}}$, can be used to assess the accuracy of the time advancement algorithm used in GENE. As observed in Fig. 1, both tests show that the algorithms used in GENE allow to satisfy the general constraints imposed by the free energy balance very satisfactorily.

The time evolution of the entropy and electrostatic energy are shown in Fig. 2. It is observed that $\mathcal{E}_{f}$ is systematically much greater than $\mathcal{E}_{\phi}$. It is also noted that both quantities rapidly reach, after a very short transient period, a statistically stationary state corresponding to saturated turbulence.

The various contributions to the evolution of $\mathcal{E}_{f}$ and $\mathcal{E}_{\phi}$ are shown in Fig. 3. It is observed that the dissipation terms are indeed pumping entropy and electrostatic energy out of the system while entropy is injected through the gradient term. The curvature term appears to transform entropy into electrostatic energy while, on the contrary, the parallel term is transforming electrostatic energy into entropy at about the same rate.

The same information is presented schematically in figures 4 and 5 . The observation that the free energy is largely dominated by its entropy part can be explained by at least two reasons. First, the entropy is the only part that is driven by the gradient term. Second, the almost perfect balance between the curvature and the parallel terms prevents a strong flux of free energy from the entropy to the electrostatic energy. As a consequence, the dissipation of electrostatic energy appears to be almost negligible when compared to the entropy dissipation $\mathcal{D}_{f} / \mathcal{D}_{\phi} \approx 400$.

The numerical dissipation terms (2) used in Gene have been implemented to avoid the use of an expensive collision operator. However, it is also possible to run the
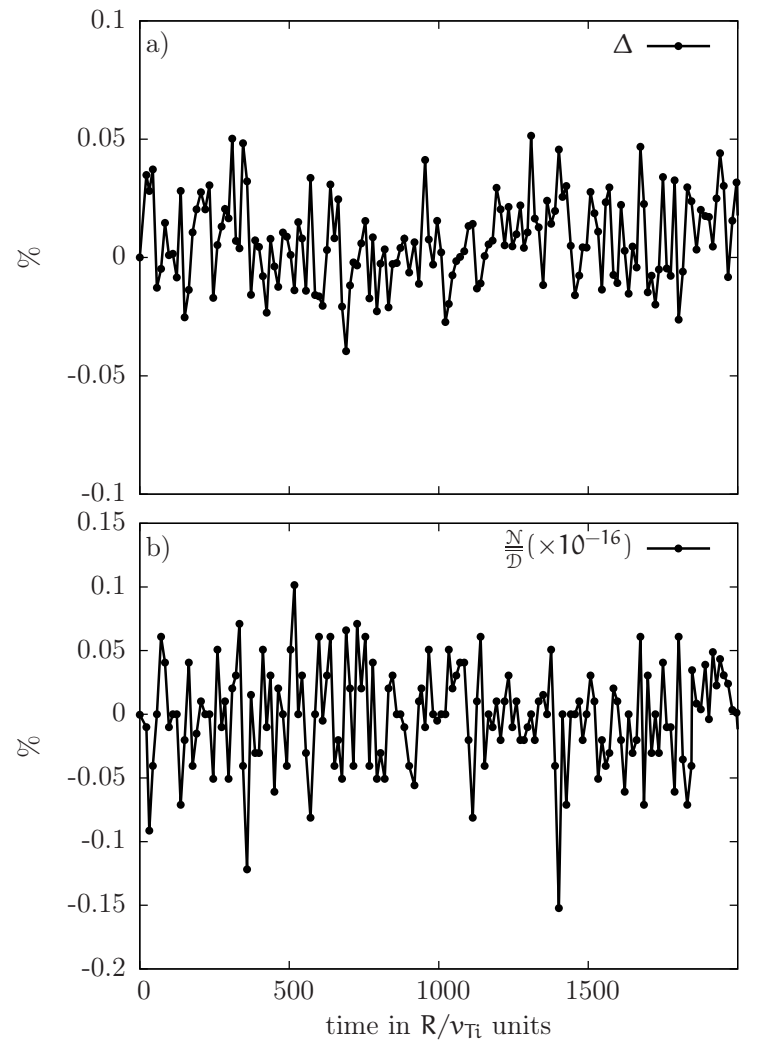

FIG. 1. (a) Plot of the residual $\Delta$ versus time. It appears that the time integration scheme satisfies the global free energy balance with a relative error systematically smaller than $510^{-4}$; (b) Conservation of the free energy balance by the nonlinear term. The curve shows that the contribution of the nonlinearity to the free energy time derivative is negligible (amounting to machine precision).

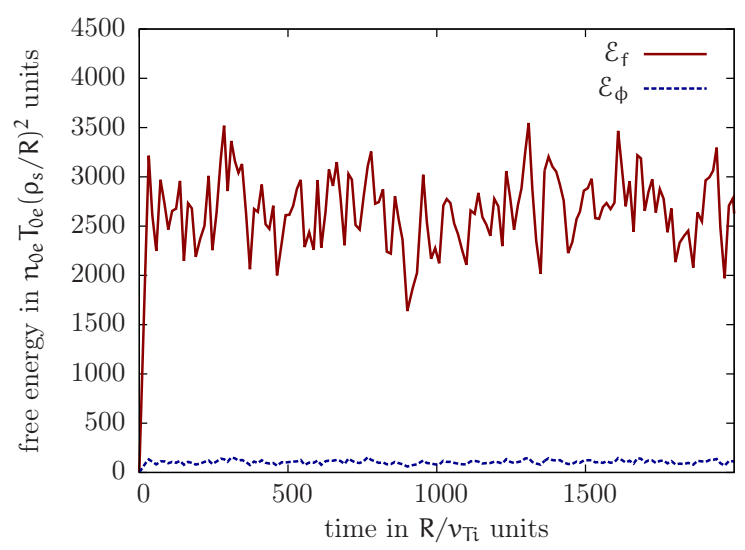

FIG. 2. Time evolution of $\mathcal{E}_{f}$ and $\mathcal{E}_{\phi}$.

code with a linerized Landau-Boltzmann collision operator. In order to check that the free energy balance is not too strongly affected by the dissipation mechanisms, runs have been performed using this collision operator with a collision frequency is $\nu\left(R / v_{T}\right)=3.0 \times 10^{-3}$ much lower than the inverse of the dynamic time scales of the sys- 


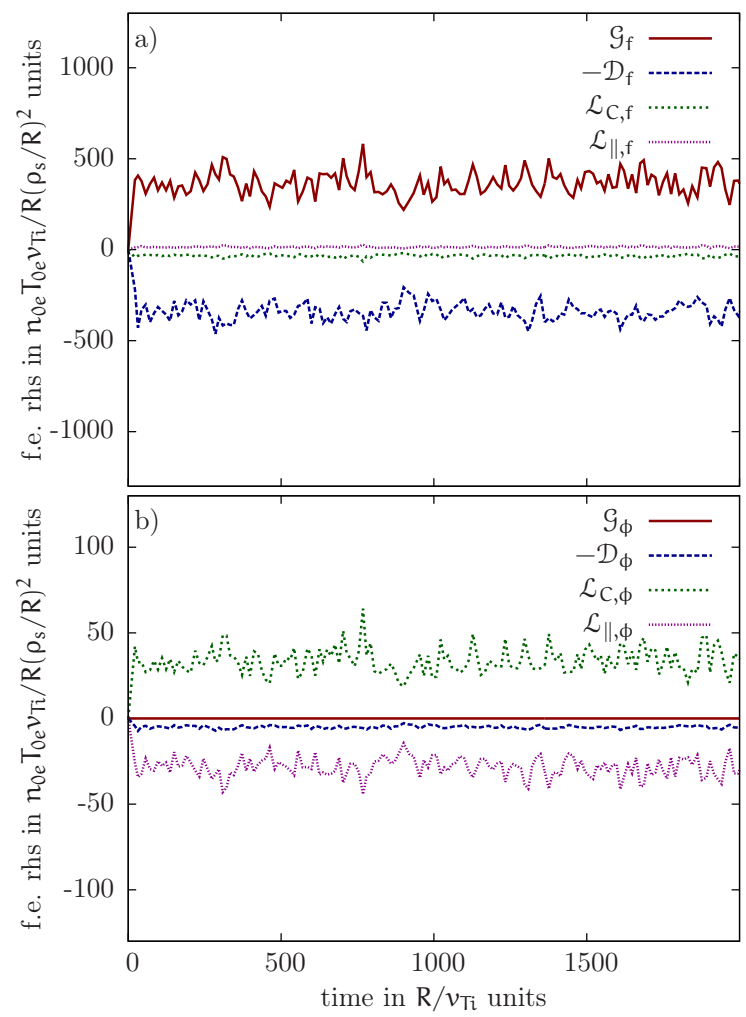

FIG. 3. Different contributions to the time derivatives of $\mathcal{E}_{f}$ (a) and $\mathcal{E}_{\phi}$ (b) versus time.

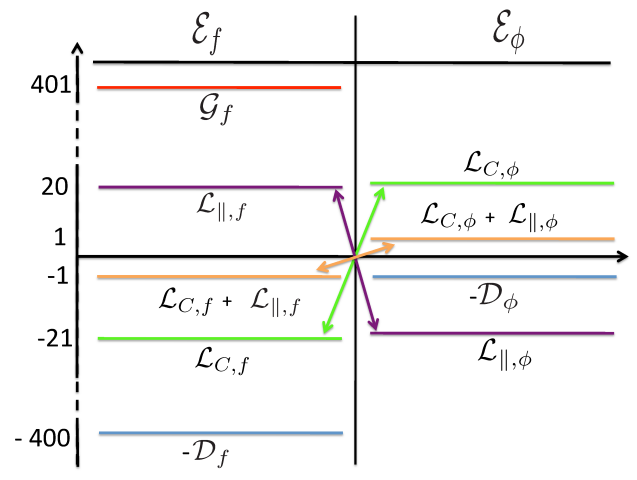

FIG. 4. Schematic plot of the different contributions of $\mathcal{E}_{f}$ and $\mathcal{E}_{\phi}$, taken from the GENE simulation described in the paper.

tem. Such a choice corresponds to the low collisionality regime. The evolution of $\mathcal{E}_{f}$ and $\mathcal{E}_{\phi}$ are then unchanged except that the dissipation terms $\mathcal{D}_{f}$ and $\mathcal{D}_{\phi}$ have to be replaced respectively by collision terms $\mathcal{C}_{f}$ and $\mathcal{C}_{\phi}$ given by

$$
\begin{aligned}
\mathcal{C}_{f} & =-\int d \Lambda \frac{T_{0 i}}{F_{0 i}} f \mathcal{C}[f] \\
\mathcal{C}_{\phi} & =-\int d \Lambda q_{i} \bar{\phi}_{1} \mathcal{C}[f]
\end{aligned}
$$

where $\mathcal{C}[f]$ is the Landau collision operator. The different

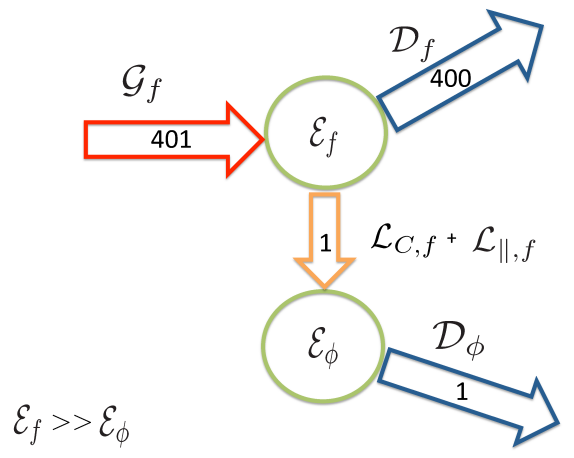

FIG. 5. Diagram of the overall free energy balance, showing the dominance of the entropy and the passive role of the electrostatic energy term.

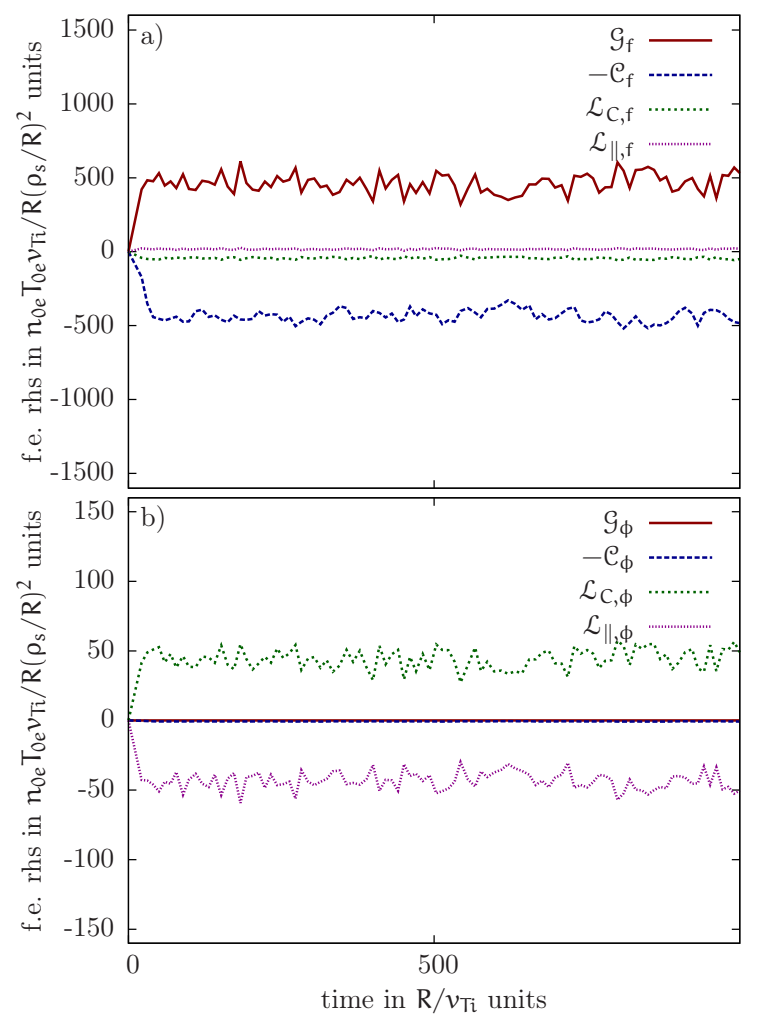

FIG. 6. Different contributions of $\mathcal{E}_{f}$ (a) and $\mathcal{E}_{\phi}$ (b) versus time with a collision operator.

terms entering the evolution equations for $\mathcal{E}_{f}$ and $\mathcal{E}_{\phi}$ are shown in Fig. 6.

It is observed that the collision operator for the entropy $\mathcal{C}_{f}$ plays the same role as $\mathcal{D}_{f}$. However, $\mathcal{C}_{\phi}$ is now almost negligible. Since a statistically stationary regime is reached, the negligible electrostatic energy collision contribution $\mathcal{C}_{\phi} \approx 0$ implies that the curvature and parallel terms have to be in balance. Except for this minor difference, there is not a significant change in the free energy balance for $\mathcal{E}_{f}$ or $\mathcal{E}_{\phi}$ when a realistic collision operator 
is used instead of numerical dissipation. The fact that $\mathcal{L}_{C, f} \approx-\mathcal{L}_{\|, f}$ when a collisional operator or dissipation term is used supports the idea that the dissipation term are doing a good job in representing the collisional effects.

\section{LOCAL FREE ENERGY BALANCE}

The global balance equations analyzed in the preceding section gives the overall picture of the fluxes of entropy and electrostatic energy in the system. However, no information is provided on the scales at which these fluxes are the most active. In order to obtain such a scale by scale information, it is necessary to introduce the Fourier representation of the free energy balance given by Eqs. $(12,13)$ in the radial and poloidal directions. Thanks to the Parseval theorem, the entropy can be rewritten as:

$$
\mathcal{E}_{f}=\int \mathrm{d} x \mathrm{~d} y \int \mathrm{d} \Theta \frac{T_{0 i}}{F_{0 i}} \frac{f^{2}}{2}=\sum_{k} \int \mathrm{d} \Theta \frac{T_{0 i}}{F_{0 i}} \frac{\left|f_{k}\right|^{2}}{2}
$$

where the sum is over all the $k_{x}$ and $k_{y}$. In the following, the spectral density of entropy will be noted

$$
\mathcal{E}_{f}^{k}=\int \mathrm{d} \Theta \frac{T_{0 i}}{F_{0 i}} \frac{\left|f_{k}\right|^{2}}{2} .
$$

Similarly, a spectral density of electrostatic energy can be defined. Remarkably, the dissipation, injection, curvature and parallel contributions to the balance equations all come from linear terms in the gyrokinetic equation (1). Their effect on balance equation can then also be split into spectral density contribution that will be noted $\mathcal{D}_{f}^{k}$, $\mathcal{D}_{\phi}^{k}, \mathcal{G}_{f}^{k}, \mathcal{L}_{C, f}^{k}$ and $\mathcal{L}_{\|, f}^{k}$. This simple mathematical property has a very important physical consequence. None of these terms can be responsible for a transfer of entropy or of electrostatic energy between different Fourier modes.

The only term that can be responsible for such transfers is the nonlinear term. Indeed, even if it does not influence the global free energy balance equations, the nonlinear term has a non-vanishing contribution on each Fourier mode of the entropy and electrostatic energy spectral densities. For instance, its contribution to the evolution of $\mathcal{E}_{f}^{k}$ is given by:

$$
\left.\frac{\partial \mathcal{E}_{f}^{k}}{\partial t}\right|_{\mathcal{N}}=\int \mathrm{d} \Theta \frac{T_{0}}{F_{0}} f_{k}^{*} N_{k}=\sum_{k^{\prime}} \mathcal{T}_{f}^{k, k^{\prime}}
$$

where $f_{k}^{*}$ is the complex conjugate value of $f_{k}$ and $N_{k}$ is the Fourier mode of the nonlinear term $\left(\left[\bar{\phi}_{1}, f\right]_{x y}\right)_{k}$. Since the product in the $x-y$ space is expressed by a convolution in the Fourier space, the following expression is easily derived:

$$
\begin{aligned}
\mathcal{T}_{f}^{k, k^{\prime}}=\int \mathrm{d} \Theta \frac{T_{0 i}}{F_{0 i}} f_{k}^{*}( & \left(k_{x}-k_{x}^{\prime}\right) \bar{\phi}_{1\left(k-k^{\prime}\right)} k_{y}^{\prime} f_{k^{\prime}} \\
& \left.-\left(k_{y}-k_{y}^{\prime}\right) \bar{\phi}_{1\left(k-k^{\prime}\right)} k_{x}^{\prime} f_{k^{\prime}}\right) .
\end{aligned}
$$

This term will be referred to as the transfer term between the mode $f_{k}$ and the mode $f_{k^{\prime}}$. Its expression comes immediately from the Poisson bracket in Fourier space. In fact, due to the Poisson equation, the electric potential $\bar{\phi}_{1\left(k-k^{\prime}\right)}$ is a linear function of the distribution $f_{k-k^{\prime}}$ and the transfer term appears to be a cubic term in $f$ involving modes $f_{k}, f_{k-k^{\prime}}$ and $f_{k^{\prime}}$. Such a so-called triadic interactions will be here interpreted as an exchange of entropy between two modes $\left(f_{k}\right.$ and $\left.f_{k^{\prime}}\right)$ because of the following important property:

$$
\mathcal{T}_{f}^{k, k^{\prime}}=-\mathcal{T}_{f}^{k^{\prime}, k} .
$$

A similar approach can be used for the nonlinear term appearing in the equation for the electrostatic energy which is given by:

$$
\left.\frac{\partial \mathcal{E}_{\phi}^{k}}{\partial t}\right|_{\mathcal{N}}=\int \mathrm{d} \Theta q_{i} \bar{\phi}_{1 k}^{*} N_{k}=\sum_{k^{\prime}} \mathcal{T}_{\phi}^{k, k^{\prime}}
$$

where

$$
\begin{aligned}
\mathcal{T}_{\phi}^{k, k^{\prime}}=\int \mathrm{d} \Theta q_{i} \bar{\phi}_{1 k}^{*}\left(k_{x}^{\prime} \bar{\phi}_{1 k^{\prime}}\left(k_{y}-k_{y}^{\prime}\right) f_{k-k^{\prime}}\right. \\
\\
\left.\quad-k_{y}^{\prime} \bar{\phi}_{1 k^{\prime}}\left(k_{x}-k_{x}^{\prime}\right) f_{k-k^{\prime}}\right) .
\end{aligned}
$$

Again, this triadic interaction will be referred to as the electrostatic energy transfer term between the two modes $\bar{\phi}_{1 k}$ and $\bar{\phi}_{1 k^{\prime}}$ because of the following property:

$$
\mathcal{T}_{\phi}^{k, k^{\prime}}=-\mathcal{T}_{\phi}^{k^{\prime}, k} .
$$

The complete Fourier representation $\mathcal{E}_{f}^{k}$ and $\mathcal{E}_{\phi}^{k}$ (including all the linear terms) then reads respectively, as

$$
\frac{\partial \mathcal{E}_{f}^{k}}{\partial t}=\sum_{k^{\prime}} T_{f}^{k, k^{\prime}}+\mathcal{G}_{f}^{k}+\mathcal{L}_{C, f}^{k}+\mathcal{L}_{\|, f}^{k}-\mathcal{D}_{f}^{k}
$$

and

$$
\begin{aligned}
\frac{\partial \mathcal{E}_{\phi}^{k}}{\partial t} & =\sum_{k^{\prime}} T_{\phi}^{k, k^{\prime}}+\mathcal{L}_{C, \phi}^{k}+\mathcal{L}_{\|, \phi}^{k}-\mathcal{D}_{\phi}^{k} \\
& =\sum_{k^{\prime}} T_{\phi}^{k, k^{\prime}}-\mathcal{L}_{C, f}^{k}-\mathcal{L}_{\|, f}^{k}-\mathcal{D}_{\phi}^{k}
\end{aligned}
$$

The different linear contributions of $\mathcal{E}_{f}^{k}$ and $\mathcal{E}_{\phi}^{k}$ (averaged over time during the saturated phase of the simulation) as a function of $k_{y}$ summed over $k_{x}$ are shown in Fig. 7.

The injection of entropy $\mathcal{G}_{f}^{k}$ appears to be well localized at low $k_{y}$. Hence, the imposed temperature gradient directly affects the largest scales of the system without noticeable effect in the smallest scales. However, dissipation is active at all scales. An explanation of this phenomenon may be provided in terms of the nonlinear coupling to damped eigenmodes ${ }^{13}$. Hence, the picture is somewhat different from the fluid turbulence cascade 

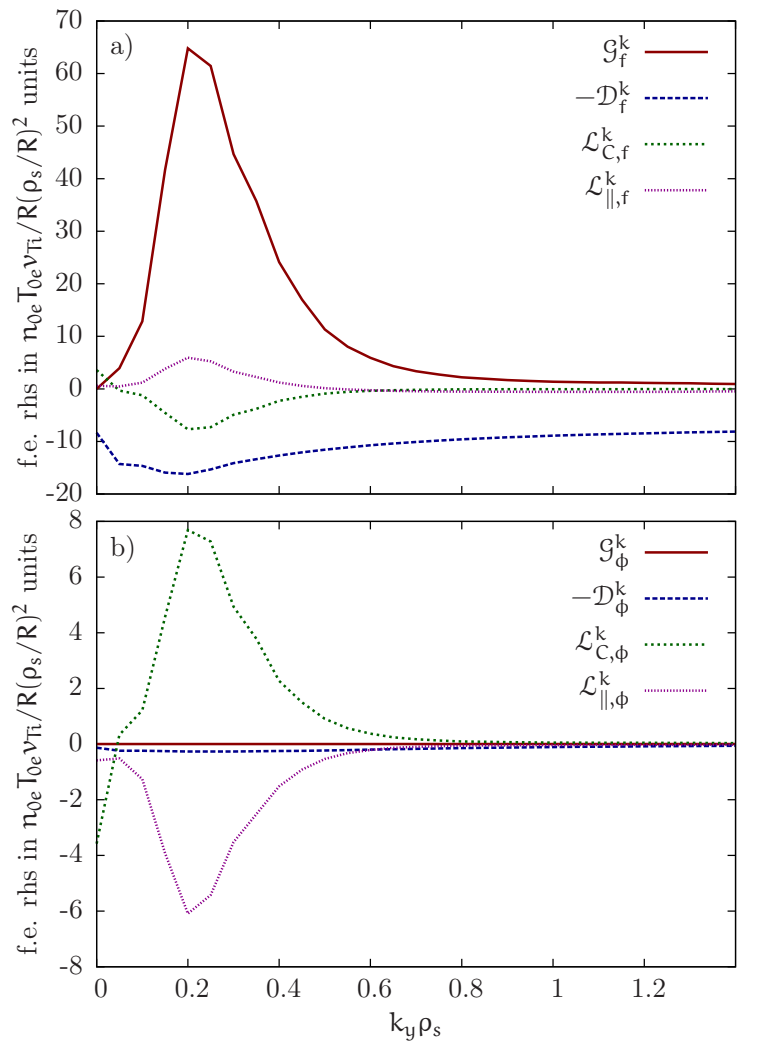

FIG. 7. Different linear contributions of $\mathcal{E}_{f}$ (a) and $\mathcal{E}_{\phi}$ (b) as a function of $k_{y}$ summed over $k_{x}$.

in which the damping term is peaked in the small scale ranges. However, the dissipation is clearly not intense enough in the large scale range to compensate exactly the entropy injection. The system has to transfer entropy towards the small scales in order to dissipate at the same rate it is injected.

The linear curvature and parallel terms appear to be important in the forcing range only and are almost always opposite to each other. The net effect of these two terms is thus almost negligible in the entropy equation. However, since there is no electrostatic energy injection, the small imbalance between these two terms is the only mechanism that act as a source of $\mathcal{E}_{\phi}^{k}$.

These curves give the net entropy and electrostatic energy injection or dissipation rates due to the various terms appearing in the local balance equations. The impact of these rates depends of course of the value of the entropy and the electrostatic energy. For this reason, it is interesting to compute a frequency associated to each term appearing in the right-hand side of Eqs. (21-22) by dividing these rates by the entropy and electrostatic energy spectral density. For instance the entropy injection scale frequency is defined by

$$
\nu_{\mathcal{G}_{f}}^{k}=\left|\frac{\mathcal{G}_{f}^{k}}{\mathcal{E}_{f}^{k}}\right| .
$$

Clearly, the dominant term in the equation will be char-
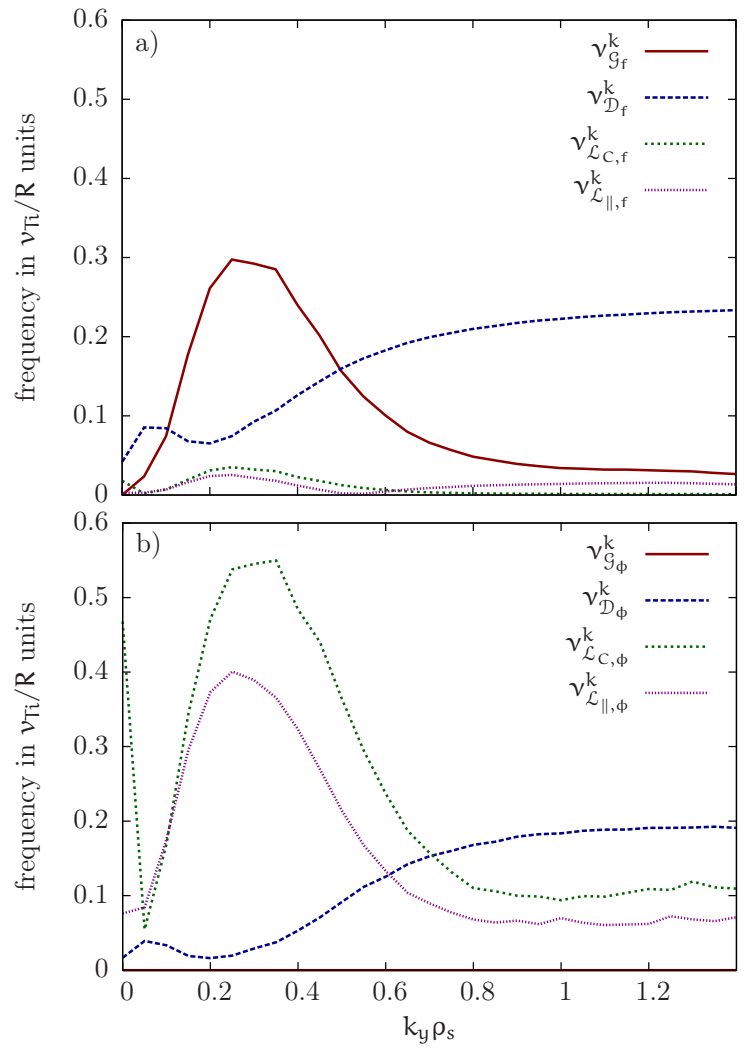

FIG. 8. Scale frequency for the different linear contributions of $\mathcal{E}_{f}(\mathrm{a})$ and $\mathcal{E}_{\phi}$ (b) as a function of $k_{y}$ summed over $k_{x}$.

acterized by the largest frequency or, equivalently, by the smallest time scale. Fig. 8 shows these frequencies as function of $k_{y}$. According to this criterion, it becomes even clearer that the entropy injection dominates at low $k_{y}$, while the entropy dissipation dominates at high $k_{y}$. The linear curvature and parallel terms appear to be characterized by the smallest frequencies at all scales in the entropy equation. In the case of the electrostatic energy equation, there is no energy injection. The linear and parallel terms appear to be dominant in the small scales, while the electrostatic energy dissipation dominates at high $k_{y}$. Similar figures are easily obtained for these quantities as function of $k_{x}$ and they show the same trends.

\section{DISCUSSION}

In the present paper, we have computed the free energy balance in a fully five-dimensional gyrokinetic simulation for a standard case of ITG turbulence. Several interesting observations can be made from this study.

First, it is observed that the free energy dynamics is largely dominated by the entropy part, while the electrostatic energy plays a passive and subdominant role. The reason is easily found in the global balance equations. Indeed, the average temperature gradient is acting as 
an "external" source of entropy, while the electrostatic energy is only driven by "internal" exchanges with the entropy.

Second, it is observed that the temperature gradients inject entropy mostly at the largest scales of the system, while the dissipation is acting throughout the entire spectrum. Moreover, an analysis of the typical frequencies as a function of the wave vectors shows that the dominant effect in the entropy balance is clearly the injection in the large scales while it is the dissipation in the small scales. No equivalent to the inertial range in NavierStokes turbulence is found here. Indeed, considering the rather limited resolution, it is not possible to identify a range of scales in which neither the injection term nor the dissipation term are active. However, since the injection and the dissipation are dominant in different ranges of wave vectors, the nonlinear term has to redistribute the entropy in a sort of cascade process.

Also, the role of the artificial dissipation has been explored. Since the results analyzed here refer to the saturated turbulent regime in which all quantities are fluctuating with time but reach statistically stationary values, the dissipation has to compensate the injection on average. When the dissipation is obtained by adding an hyper-diffusion term, its effect on the electrostatic energy is very small (more than two orders of magnitude smaller than its effect on entropy). Such a property for the artificial dissipation used mostly for improving the speed performances of the code is reassuring. Indeed, as shown on Fig. 6, a realistic collision operator barely affects the electrostatic energy and this property is thus quite well reproduced by the hyper-diffusion term.

Finally, it is also observed that the parallel and curvature terms do not play a dominant role in the entropy equation, independently of the wave vector. On the contrary, these two terms are the only contributions to the electrostatic energy balance. Although they are of opposite sign, they both appear to act mostly in the same large scale range. So, a strong cascade process of electrostatic energy cannot be triggered by these terms.

\section{ACKNOWLEDGEMENTS}

The authors would like to thank G. Plunk and D. Hatch for very fruitful discussions, and acknowledge computational resources offered by the HPC-FF supercomputer at Jülich, Germany. This work has been supported by the contract of association EURATOM - Belgian state.

${ }^{1}$ A. J. Brizard and T. S. Hahm, Rev. Mod. Phys. 79, 421 (2007).

${ }^{2}$ A. A. Schekochihin, S. C. Cowley, W. Dorland, G. W. Hammett, G. G. Howes, E. Quataert and T. Tatsuno, J. Astrophys. 182, 310 (2009).

${ }^{3}$ A. Bañón Navarro, P. Morel, M. Albrecht-Marc, D. Carati, F. Merz, T. Görler and F. Jenko, Phys. Rev. Lett. 106, 055001 (2011).

${ }^{4}$ G. Falkovich and K.R. Sreenivasan, Physics Today 59, 43 (2006)

${ }^{5}$ X. Lapillone, S. Brunner, T. Dannert, S. Jolliet, A. Marinoni, L. Villard, T. Görler, F. Jenko and F. Merz, Phys. Plasmas 16, 032308 (2009).

${ }^{6}$ F. Merz, PhD Thesis, Universität Münster, 2009.

${ }^{7}$ F. Jenko, W. Dorland, M. Kotschenreuther and B. N. Rogers, Phys. Plasmas 7, No. 5, 1904-1910 (2000).

${ }^{8}$ T. Dannert, F. Jenko, Phys. Plasmas 12, 072309 (2005).

${ }^{9}$ M. J. Pueschel, T. Dannert and F. Jenko, Comp. Phys. Comm. 181, 1428 (2010).

${ }^{10}$ T. Tatsuno, M. Barnes, S. C. Cowley, W. Dorland, G. G. Howes, R. Numata, G. G. Plunk and A. A. Schekochihin, J. Plasma Fusion Res. SERIES 9, 509 (2010).

${ }^{11}$ J. Candy and R. E. Waltz, Phys. Plasmas, 13, 032310 (2006).

${ }^{12}$ A. M. Dimits, G. Bateman, M. A. Beer, B. I. Cohen, W. Dorland, G. W. Hammett, C. Kim, J. E. Kinsey, M. Kotschenreuther, A. H. Kritz, L. L. Lao, J. Mandrekas, W. M. Nevins, S. E. Parker, A. J. Redd, D. E. Shumaker, R. Sydora and J. Weiland, Phys. Plasmas 7, 969 (2000).

${ }^{13}$ D. R. Hatch, P. W. Terry, F. Jenko, F. Merz and W. M. Nevins, Phys. Rev. Lett. 106, 115003 (2011). 